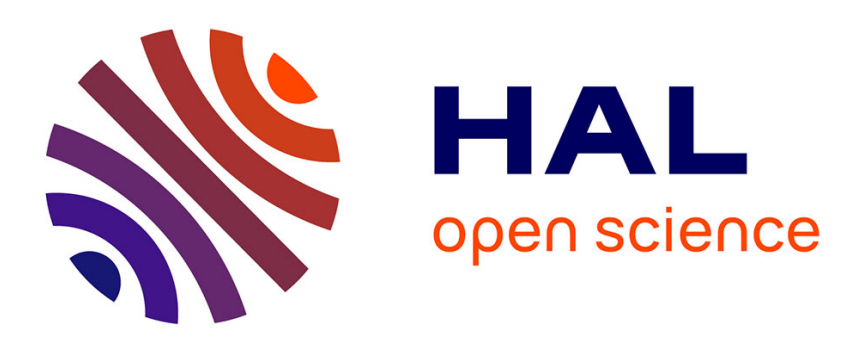

\title{
Adaptive sliding mode control for quadrotor attitude stabilization and altitude tracking
}

Hakim Bouadi, Sebastião Simoes Cunha, Antoine Drouin, Felix Mora-Camino

\section{To cite this version:}

Hakim Bouadi, Sebastião Simoes Cunha, Antoine Drouin, Felix Mora-Camino. Adaptive sliding mode control for quadrotor attitude stabilization and altitude tracking. CINTI 2011, IEEE 12th International Symposium on Computational Intelligence and Informatics, Nov 2011, Budapest, Hungary. pp 449 - 455, 10.1109/CINTI.2011.6108547 . hal-00938518

\section{HAL Id: hal-00938518 \\ https://hal-enac.archives-ouvertes.fr/hal-00938518}

Submitted on 29 Apr 2014

HAL is a multi-disciplinary open access archive for the deposit and dissemination of scientific research documents, whether they are published or not. The documents may come from teaching and research institutions in France or abroad, or from public or private research centers.
L'archive ouverte pluridisciplinaire HAL, est destinée au dépôt et à la diffusion de documents scientifiques de niveau recherche, publiés ou non, émanant des établissements d'enseignement et de recherche français ou étrangers, des laboratoires publics ou privés. 


\title{
Adaptive Sliding Mode Control for Quadrotor Attitude Stabilization and Altitude Tracking
}

\author{
Hakim Bouadi, S. Simoes Cunha, A. Drouin and F. Mora-Camino
}

\begin{abstract}
Adaptive control algorithms are of interest in flight control systems design not only for their capability to improve performance and reliability but also for handling aerodynamic parameter uncertainties, external disturbances and modeling inaccuracies. In this paper, a direct adaptive sliding mode control is developed for the quadrotor attitude stabilization and altitude trajectory tracking. First, developed controller is applied without considering disturbances and parameter uncertainties. After, a centered white gaussian noise with some parameter uncertainties are added to the considered output vector, mass and inertia matrix, respectively. The synthesis of the adaptation laws is based on the positivity and Lyapunov design principle. Numerical simulations are performed showing the robustness of the proposed control technique.
\end{abstract}

Index Terms-feedback linearization, adaptive control, adaptation mechanism, positivity principle, sliding mode.

\section{INTRODUCTION}

$\mathbf{U}$ NMANNED aerial vehicles (UAV) have known a growing interest thanks to recent technological advances, especially those related to instrumentation which turn possible the design of powerful systems (mini drones) endowed with real capacities of autonomous navigation at reasonable cost [1].

These flying devices have been used in many civilian and military applications while new configurations and control laws should be developed to insure their applicability to new mission under more adverse environments. A special class of UAVs, called quadrotors, has been studied recently by some authors [1], [2], [3], [4], [5], [6]-[7]. These systems as many other dynamic systems, present constant or slowly-varying uncertain parameters.

Adaptive control provides many tools to tackle such systems. The basic idea in adaptive control is to estimate the uncertain plant parameters or, equivalently, is to perform an on-line estimation of the corresponding controller parameters

H. Bouadi is PhD student and he is with the Automation and Operational Research Laboratory (LARA) in the National Civil Aviation InstituteENAC. 07, Avenue Edouard Belin, 31400-Toulouse-France (phone: +33 (0)-601-18-83-91, fax: +33 (0)-5-62-17-40-23 hakimbouadi@yahoo. fr, Hakim. Bouadidenac.fr.

S. Simoes Cunha is Associated Professor at Federal University of Itajuba Brazil, Mechanical Engineering Institute, he has a post-doctoral position at ENAC LARA, funded by CAPES sebas aunifei.edu.br

A. Drouin is $\mathrm{PhD}$ student and he is with the Automation and Operational Research Laboratory (LARA) in the National Civil Aviation Institute-ENAC poinix@gmail.com

F. Mora-Camino is Professor at the National Civil Aviation Institute and he is the director of the Automation and Operational Research Laboratory (LARA) at Air Transportation Department moracaminoehotmail.fr.

Manuscript received September 30, 2011.This work was supported by Automation and Operational Research Laboratory of Air Transportation Department in the National Civil Aviation institute in Toulouse-France. based on the measured system signals, and to use the estimated parameters in the control input computation [8], [9], [10]-[11].

In this paper, The control of a quadrotor flying in an environment subject to atmospheric perturbation is considered where the controllability condition is checked. First, a dynamic modeling of a quadrotor is considered taking into account various parameters which can affect the dynamics of a flying structure such as frictions due to the aerodynamic torques, drag forces along $(x, y, z)$ axis and gyroscopic effects. Then, an adaptive sliding mode controller is synthesized in order to stabilize both bank and pitch angles while tracking heading and altitude trajectories and to compensate additive perturbation and parameter uncertainties related to the mass and inertia matrix of the quadrotor. Adaptation laws are developed based-on Lyapunov approach where the asymptotic stability is guaranteed. Finally, numerical simulations are displayed, showing acceptable results for the proposed control solution.

\section{Flight Dynamics Modeling}

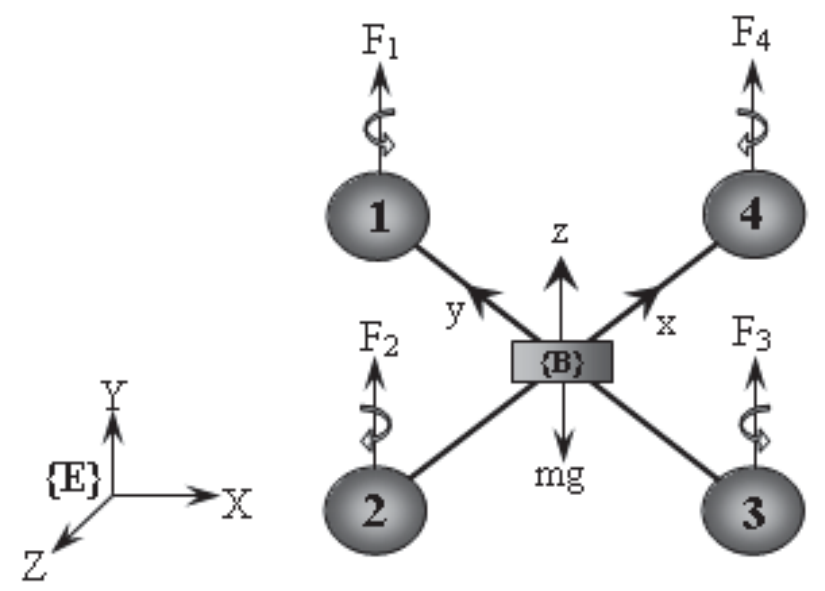

Fig. 1. Quadrotor Configuration

The quadrotor has four propellers in cross configuration where two pairs of propellers turn in opposite directions. By varying the rotor speeds, one can change the lift forces and create motion. Thus, increasing or decreasing evenly the four propellers speeds together generates vertical motion.

Changing evenly the 2 and 4 propellers speed conversely produces roll rotation coupled with lateral motion.

Pitch rotation and the corresponding lateral motion result from 1 and 3 propellers speed conversely modified. Yaw rotation is more subtle, as it results from the difference in the counter-torque between each pair of propellers. 
To limit the complexity of the dynamics modeling, the following assumptions are adopted [1]:

1. The quadrotor structure is rigid and symmetrical

2. The propellers are rigid

3. Thrust and drag forces are proportional to the square of propellers speed rotation.

Under these assumptions, it is possible to describe its flight dynamics as that of a rigid body under aerodynamic forces and moments caused by the propellers rotation. Using the formalism of Newton-Euler, the dynamics equations are written in the following way [1]:

$$
\begin{gathered}
m \ddot{\zeta}=F_{f}+F_{d}+F_{g} \\
J \dot{\Omega}=-\Omega \wedge J \Omega+\Gamma_{f}-\Gamma_{a}-\Gamma_{g}
\end{gathered}
$$

where $\zeta$ is the position of the quadrotor centre of mass with respect to the inertial frame $E, m$ is the total mass of the structure and $J \in \mathbb{R}^{3 \times 3}$ is a symmetric positive definite constant inertia matrix of the quadrotor with respect to the body fixed frame.

$$
J=\left(\begin{array}{ccc}
I_{x} & 0 & 0 \\
0 & I_{y} & 0 \\
0 & 0 & I_{z}
\end{array}\right)
$$

$\Omega$ represents the angular velocity of the quadrotor expressed in the body fixed frame such as:

$$
\Omega=\left(\begin{array}{ccc}
1 & 0 & -\sin \theta \\
0 & \cos \phi & \cos \theta \sin \phi \\
0 & -\sin \phi & \cos \phi \cos \theta
\end{array}\right)\left(\begin{array}{c}
\dot{\phi} \\
\dot{\theta} \\
\dot{\psi}
\end{array}\right)
$$

where $\phi, \theta$ and $\psi$ are bank, pitch and heading angles, respectively.

In the case when the quadrotor performs many angular motions of low amplitude, $\Omega$ can be assimilated to $[\dot{\phi}, \dot{\theta}, \dot{\psi}]^{T}$. $F_{f}$ denotes the resultant of the forces generated by the four rotors such as:

$$
F_{f}=\left(\begin{array}{c}
\cos \phi \cos \psi \sin \theta+\sin \phi \sin \psi \\
\cos \phi \sin \theta \sin \psi-\sin \phi \cos \psi \\
\cos \phi \cos \theta \\
F_{i}=K_{p} \omega_{i}^{2}
\end{array}\right) \sum_{\mathbf{i}=\mathbf{1}}^{4} F_{i}
$$

where $K_{p}$ is a coefficient related to the lift force and $\omega_{i}$ is the angular rotor speed. $F_{d}$ denotes the resultant of the drag forces along $(X, Y, Z)$ axis such as:

$$
F_{d}=\left(\begin{array}{ccc}
-K_{f d x} & 0 & 0 \\
0 & -K_{f d y} & 0 \\
0 & 0 & -K_{f d z}
\end{array}\right) \dot{\zeta}
$$

where $K_{f d x}, K_{f d y}$ and $K_{f d z}$ are positive translation drag coefficients. $F_{g}$ is the gravity force and it is expressed as follows:

$$
F_{g}=\left(\begin{array}{c}
0 \\
0 \\
-m g
\end{array}\right)
$$

$\Gamma_{f}$ is the moment developed by the quadrotor according to the body fixed frame such as:

$$
\Gamma_{f}=\left(\begin{array}{c}
d\left(F_{3}-F_{1}\right) \\
d\left(F_{4}-F_{2}\right) \\
C_{D}\left(\omega_{1}^{2}-\omega_{2}^{2}+\omega_{3}^{2}-\omega_{4}^{2}\right)
\end{array}\right)
$$

$d$ is the distance between the quadrotor centre of mass and the rotation axis of propeller and $C_{D}$ is the drag coefficient. $\Gamma_{a}$ is the resultant of aerodynamic frictions torques such as:

$$
\Gamma_{a}=\left(\begin{array}{ccc}
K_{f a x} & 0 & 0 \\
0 & K_{f a y} & 0 \\
0 & 0 & K_{f a z}
\end{array}\right)\|\Omega\|^{2}
$$

where $K_{f a x}, K_{f a y}$ and $K_{f a z}$ are the coefficients of aerodynamic frictions. $\Gamma_{g}$ is the resultant of torques due to the gyroscopic effects:

$$
\Gamma_{g}=\sum_{i=1}^{4} \Omega \wedge J_{r}\left(\begin{array}{c}
0 \\
0 \\
(-1)^{i+1} \omega_{i}
\end{array}\right)
$$

where $J_{r}$ represents the rotor inertia.

Consequently the complete dynamic model which governs the quadrotor is as follows:

$$
\begin{gathered}
\ddot{\phi}=\frac{1}{I_{x}}\left[\left(I_{y}-I_{z}\right) \dot{\psi} \dot{\theta}-K_{f a x} \dot{\phi}^{2}-J_{r} \bar{\Omega} \dot{\theta}+d U_{2}\right] \\
\ddot{\theta}=\frac{1}{I_{y}}\left[\left(I_{z}-I_{x}\right) \dot{\psi} \dot{\phi}-K_{f a y} \dot{\theta}^{2}+J_{r} \bar{\Omega} \dot{\phi}+d U_{3}\right] \\
\ddot{\psi}=\frac{1}{I_{z}}\left[\left(I_{x}-I_{y}\right) \dot{\phi} \dot{\theta}-K_{f a z} \dot{\psi}^{2}+C_{D} U_{4}\right] \\
\ddot{x}=\frac{1}{m}\left[-K_{f d x} \dot{x}+(\cos \phi \sin \theta \cos \psi+\sin \phi \sin \psi) U_{1}\right] \\
\ddot{y}=\frac{1}{m}\left[-K_{f d y} \dot{y}+(\cos \phi \sin \theta \sin \psi-\sin \phi \cos \psi) U_{1}\right] \\
\ddot{z}=\frac{1}{m}\left[-K_{f d z} \dot{z}+(\cos \phi \cos \theta) U_{1}\right]-g
\end{gathered}
$$

where $U_{1}, U_{2}, U_{3}$ and $U_{4}$ are the control inputs of the system which are written according to the angular velocities of the four rotors as follows:

$$
\begin{gathered}
\left(\begin{array}{c}
U_{1} \\
U_{2} \\
U_{3} \\
U_{4}
\end{array}\right)=\left(\begin{array}{cccc}
K_{p} & K_{p} & K_{p} & K_{p} \\
-K_{p} & 0 & K_{p} & 0 \\
0 & -K_{p} & 0 & K_{p} \\
C_{D} & -C_{D} & C_{D} & -C_{D}
\end{array}\right)\left(\begin{array}{c}
\omega_{1}^{2} \\
\omega_{2}^{2} \\
\omega_{3}^{2} \\
\omega_{4}^{2}
\end{array}\right) \\
\bar{\Omega}=\omega_{1}-\omega_{2}+\omega_{3}-\omega_{4}
\end{gathered}
$$

The dynamic modeling developed above (10) is completed by the following control inputs constraints:

$$
\begin{gathered}
0 \leqslant U_{1} \leqslant 4 K_{p} \omega_{\text {max }}^{2} \\
-K_{p} \omega_{\text {max }}^{2} \leqslant U_{2} \leqslant K_{p} \omega_{\text {max }}^{2} \\
-K_{p} \omega_{\text {max }}^{2} \leqslant U_{3} \leqslant K_{p} \omega_{\text {max }}^{2} \\
-2 C_{D} \omega_{\text {max }}^{2} \leqslant U_{4} \leqslant 2 C_{D} \omega_{\text {max }}^{2}
\end{gathered}
$$

The rotor is a unit constituted by D.C-motor actuating a propeller via a reducer. The D.C-motor is governed by the following dynamics:

$$
\begin{gathered}
V=R I+L \frac{d I}{d t}+K_{e} \omega \\
K_{m} I=J_{r} \frac{d \omega}{d t}+K_{r} \omega^{2}+C_{s}
\end{gathered}
$$


where $V$ is the motor input tension. $K_{e}$ and $K_{m}$ represent the electrical and mechanical torque constant, respectively. $K_{r}$ is the load constant torque. $R$ and $J_{r}$ are the motor internal resistance and the rotor inertia, respectively. $C_{s}$ denotes the solid friction.

Then the model which characterizes the rotor dynamics is:

$$
\dot{\omega}_{i}=b V+\beta_{0}+\beta_{1} \omega_{i}+\beta_{2} \omega_{i}^{2}
$$

with:

$$
\beta_{0}=\frac{-C_{s}}{J_{r}} \quad \beta_{1}=\frac{-K_{e} K_{m}}{R J_{r}} \quad \beta_{2}=\frac{-K_{r}}{J_{r}} \quad b=\frac{K_{m}}{R J_{r}}
$$

\section{Control Design With Parameter Uncertainty}

To synthesize adaptive laws, it is assumed that some uncertainty remains with respect to the main coefficients related to the aerodynamic torques, inertia matrix and mass of the system. The main control objective is here the stabilization of bank and pitch angles while tracking heading and altitude trajectories. In order to achieve safely this control objective a nonlinear adaptive sliding mode control is developed.

Let $X=[\phi, \dot{\phi}, \theta, \dot{\theta}, \psi, \dot{\psi}, z, \dot{z}]^{T}$ and $U=\left[U_{2}, U_{3}, U_{4}, U_{1}\right]^{T}$ be the state and the control input vectors, respectively. The equations (10) can be written under the following affine state representation such as:

$$
\begin{gathered}
\dot{X}=f(X)+g(X) U \\
x_{2} \\
(X)=\left(\begin{array}{c}
a_{1} x_{4} x_{6}+a_{2} x_{2}^{2}+a_{3} \bar{\Omega} x_{4} \\
x_{4} \\
a_{4} x_{2} x_{6}+a_{5} x_{4}^{2}+a_{6} \bar{\Omega} x_{2} \\
x_{6} \\
a_{7} x_{2} x_{4}+a_{8} x_{6}^{2} \\
x_{8} \\
a_{9} x_{8}-g
\end{array}\right)
\end{gathered}
$$

and $g(X)=\left[g_{1}, g_{2}, g_{3}, g_{4}\right]$ represents the control matrix:

$$
g(X)=\left(\begin{array}{cccc}
0 & 0 & 0 & 0 \\
b_{1} & 0 & 0 & 0 \\
0 & 0 & 0 & 0 \\
0 & b_{2} & 0 & 0 \\
0 & 0 & 0 & 0 \\
0 & 0 & b_{3} & 0 \\
0 & 0 & 0 & 0 \\
0 & 0 & 0 & \frac{\cos x_{1} \cos x_{3}}{m}
\end{array}\right)
$$

with:

$$
\begin{array}{lllll}
a_{1}=\frac{I_{y}-I_{z}}{I_{x}} & a_{2}=\frac{-K_{f a x}}{I_{x}} & a_{3}=\frac{-J_{r}}{I_{x}} & a_{4}=\frac{I_{z}-I_{x}}{I_{y}} \\
a_{5}=\frac{-K_{f a y}}{I_{y}} & a_{6}=\frac{J_{r}}{I_{y}} & a_{7}=\frac{I_{x}-I_{y}}{I_{z}} & a_{8}=\frac{-K_{f a z}}{I_{z}} \\
a_{9}=\frac{-K_{f d z}}{m} & b_{1}=\frac{d}{I_{x}} & b_{2}=\frac{d}{I_{y}} & b_{3}=\frac{C_{D}}{I_{z}}
\end{array}
$$

The controllability matrix $\Upsilon$ for the system (15a) is given by:

$$
\Upsilon=\left[g_{1}, g_{2}, g_{3}, g_{4}, a d_{f} g_{1}, a d_{f} g_{2}, a d_{f} g_{3}, a d_{f} g_{4}\right]
$$

where $a d_{f} g_{i}$ represents the Lie brackets such as:

$$
\begin{gathered}
a d_{f} g_{i}=\nabla g_{i} f-\nabla f g_{i} \\
\operatorname{det}(\Upsilon)=\frac{b_{1}^{2} b_{2}^{2} b_{3}^{2} \cos x_{1}^{2} \cos x_{3}^{2}}{m^{2}}
\end{gathered}
$$

It can be concluded that $\forall\left(x_{1}, x_{3}\right) \in \mathbb{R}^{2}$ such that $\left(x_{1}, x_{3} \neq\right.$ $\left.\frac{\sqrt{2 \pi}}{2}\right)$ and $\forall\left(b_{1}, b_{2}, b_{3}\right) \in \mathbb{R}^{* 3}$, the considered above system (15a) is locally controllable over $\mathbb{R}^{8}$.

Let $y=[\phi, \theta, \psi, z]^{T}$ be the output vector. The relative degree of the considered system is equal to 8 since:

$$
\begin{gathered}
\ddot{y}_{1}=a_{1} x_{4} x_{6}+a_{2} x_{2}^{2}+a_{3} \bar{\Omega} x_{4}+b_{1} U_{2} \\
\ddot{y}_{2}=a_{4} x_{2} x_{6}+a_{5} x_{4}^{2}+a_{6} \bar{\Omega} x_{2}+b_{2} U_{3} \\
\ddot{y}_{3}=a_{7} x_{2} x_{4}+a_{8} x_{6}^{2}+b_{3} U_{4} \\
\ddot{y}_{4}=a_{9} x_{8}+\frac{\cos x_{1} \cos x_{3}}{m} U_{1}-g
\end{gathered}
$$

then, no internal dynamics is associated with this output vector. The equation (20a) can be rewritten under a linearly parameterized form such as:

$$
U_{2}=h_{1} \ddot{y}_{1}-\lambda_{1} x_{4} x_{6}-\lambda_{2} x_{2}^{2}-\lambda_{3} \bar{\Omega} x_{4}
$$

with:

$$
h_{1}=\frac{1}{b_{1}} \quad \lambda_{1}=\frac{a_{1}}{b_{1}} \quad \lambda_{2}=\frac{a_{2}}{b_{1}} \quad \lambda_{3}=\frac{a_{3}}{b_{1}}
$$

To synthesize the adaptive control law $U_{2}$, it is assumed that the full state vector components are available through the measure and that the sign of $h_{1}$ is known [12]. We assume also that the parameters $h_{1}$ and $\lambda_{i}$ are unknown constants.

Now, let us choose a sliding surface $\sigma_{1}$ such as:

$$
\begin{gathered}
\sigma_{1}=\dot{y}_{1}-v_{1} \\
v_{1}=\dot{\phi}_{d}-k_{1} \epsilon_{\phi} \\
\epsilon_{\phi}=\phi-\phi_{d}
\end{gathered}
$$

where $\epsilon_{\phi}$ represents the tracking error, $\phi_{d}$ is the desired bank angle and $k_{1}$ denotes a real positive parameter. Now a candidate Lyapunov positive definite function $V_{1}\left(\sigma_{1}\right)$ is defined:

$$
\begin{gathered}
V_{1}\left(\sigma_{1}\right)=\frac{1}{2} \sigma_{1}^{2} \\
\dot{V}_{1}\left(\sigma_{1}\right)=\sigma_{1}\left[\frac{1}{h_{1}}\left(U_{2}+\lambda_{1} x_{4} x_{6}+\lambda_{2} x_{2}^{2}+\lambda_{3} \bar{\Omega} x_{4}\right)-\dot{v}_{1}\right]
\end{gathered}
$$

then, if $\dot{V}_{1}\left(\sigma_{1}\right)=\sigma_{1} \dot{\sigma}_{1} \leqslant 0$, asymptotic Lyapunov stability will be guaranteed. For that the control law could be chosen as:

$$
U_{2}=\hat{h}_{1}\left[\ddot{\phi}_{d}-k_{1} \dot{\epsilon}_{\phi}-k \sigma_{1}\right]-\hat{\lambda}_{1} x_{4} x_{6}-\hat{\lambda}_{2} x_{2}^{2}-\hat{\lambda}_{3} \bar{\Omega} x_{4}
$$

since the parameters $\lambda_{1}, \lambda_{2}, \lambda_{3}$ and $h_{1}$ are unknown, so they are replaced by their estimates in the control law (24) and $k$ is a real positive parameter.

By replacing the synthesized control law expression (24) in the time derivative equation of the sliding surface $\sigma_{1}$, the closed loop dynamics can be written such as:

$$
h_{1} \dot{\sigma}_{1}+k \sigma_{1}=\tilde{\lambda}_{1} x_{4} x_{6}+\tilde{\lambda}_{2} x_{2}^{2}+\tilde{\lambda}_{3} \bar{\Omega} x_{4}-\tilde{h}_{1}\left[\ddot{\phi}_{d}-k_{1} \dot{\epsilon}_{\phi}\right]
$$


where $\tilde{\lambda}_{i}$ and $\tilde{h}_{1}$ represent the estimation errors related to the controller parameters:

$$
\begin{aligned}
\tilde{\lambda}_{i} & =\lambda_{i}-\hat{\lambda}_{i} \\
\tilde{h}_{1} & =h_{1}-\hat{h}_{1}
\end{aligned}
$$

The closed-loop dynamics (25) shows that if the estimation errors related to the controller parameters converge to zero, then the tracking error dynamics tend to zero exponentially as shown:

$$
h_{1} \dot{\sigma}_{1}+k \sigma_{1}=0
$$

To synthesize an adaptation mechanism, the positivity and Lyapunov design principle is applied in order to determine the adaptation laws which allow the on-line estimation of the unknown controller parameters $\hat{h}_{1}$ and $\hat{\lambda}_{i}$. Consequently another Lyapunov positive definite function $V_{2}\left(\sigma_{1}, \tilde{\lambda}_{i}, \tilde{h}_{1}\right)$ is introduced:

$$
V_{2}\left(\sigma_{1}, \tilde{\lambda}_{i}, \tilde{h}_{1}\right)=\frac{1}{2}\left|h_{1}\right| \sigma_{1}^{2}+\frac{1}{2 \gamma_{1}}\left[\tilde{h}_{1}^{2}+\sum_{i=1}^{3} \tilde{\lambda}_{i}^{2}\right]
$$

where $\gamma_{1}$ denotes the gain adaptation. The idea is to choose $\dot{\hat{h}}_{1}$ and $\dot{\hat{\lambda}}_{i}$ such that $\dot{V}_{2}\left(\sigma_{1}, \tilde{\lambda}_{i}, \tilde{h}_{1}\right) \leqslant 0$. Since:

$$
\begin{aligned}
\dot{V}_{2}\left(\sigma_{1}, \tilde{h}_{1}, \tilde{\lambda}_{i}\right)= & \sigma_{1} \frac{\left|h_{1}\right|}{h_{1}}\left[\tilde{\lambda}_{1} x_{4} x_{6}+\tilde{\lambda}_{2} x_{2}^{2}+\tilde{\lambda}_{3} \bar{\Omega} x_{4}\right. \\
& \left.-h_{1}\left(\ddot{\phi}_{d}-k_{1} \dot{\epsilon}_{\phi}\right)-k \sigma_{1}\right] \\
& -\frac{1}{\gamma_{1}}\left[\tilde{h}_{1} \dot{\hat{h}}_{1}+\sum_{i=1}^{3} \tilde{\lambda}_{i} \dot{\hat{\lambda}}_{i}\right]
\end{aligned}
$$

choosing the adaptation laws $\dot{\hat{h}}_{1}$ and $\dot{\hat{\lambda}}_{i}$ such as:

$$
\begin{gathered}
\dot{\hat{\lambda}}_{1}=\gamma_{1} \operatorname{sgn}\left(h_{1}\right) \sigma_{1} x_{4} x_{6} \\
\dot{\hat{\lambda}}_{2}=\gamma_{1} \operatorname{sgn}\left(h_{1}\right) \sigma_{1} x_{2}^{2} \\
\dot{\hat{\lambda}}_{3}=\gamma_{1} \operatorname{sgn}\left(h_{1}\right) \sigma_{1} \bar{\Omega} x_{4} \\
\dot{\hat{h}}_{1}=-\gamma_{1} \operatorname{sgn}\left(h_{1}\right) \sigma_{1}\left(\ddot{\phi}_{d}-k_{1} \dot{\epsilon}_{\phi}\right)
\end{gathered}
$$

implies that:

$$
\dot{V}_{2}\left(\sigma_{1}, \tilde{h}_{1}, \tilde{\lambda}_{i}\right)=-|k| \sigma_{1}{ }^{2}
$$

and the global tracking convergence of the adaptive control system is guaranteed.

The next control and adaptation laws are obtained by following the same steps presented above, therefore:

$$
U_{3}=\hat{h}_{2}\left[\ddot{\theta}_{d}-k_{2} \dot{\epsilon}_{\theta}-k^{\prime} \sigma_{2}\right]-\hat{\lambda}_{4} x_{2} x_{6}-\hat{\lambda}_{5} x_{4}^{2}-\hat{\lambda}_{6} \bar{\Omega} x_{2}
$$

with:

$$
\begin{gathered}
\dot{\hat{\lambda}}_{4}=\gamma_{2} \operatorname{sgn}\left(h_{2}\right) \sigma_{2} x_{2} x_{6} \\
\dot{\hat{\lambda}}_{5}=\gamma_{2} \operatorname{sgn}\left(h_{2}\right) \sigma_{2} x_{4}^{2} \\
\dot{\hat{\lambda}}_{6}=\gamma_{2} \operatorname{sgn}\left(h_{2}\right) \sigma_{2} \bar{\Omega} x_{2} \\
\dot{\hat{h}}_{2}=-\gamma_{2} \operatorname{sgn}\left(h_{2}\right) \sigma_{2}\left(\ddot{\theta}_{d}-k_{2} \dot{\epsilon}_{\theta}\right)
\end{gathered}
$$

and

$$
U_{4}=\hat{h}_{3}\left[\ddot{\psi}_{d}-k_{3} \dot{\epsilon}_{\psi}-k^{\prime \prime} \sigma_{3}\right]-\hat{\lambda}_{7} x_{2} x_{4}-\hat{\lambda}_{8} x_{6}^{2}
$$

with:

$$
\begin{gathered}
\dot{\hat{\lambda}}_{7}=\gamma_{3} \operatorname{sgn}\left(h_{3}\right) \sigma_{3} x_{2} x_{4} \\
\dot{\hat{\lambda}}_{8}=\gamma_{3} \operatorname{sgn}\left(h_{3}\right) \sigma_{3} x_{6}^{2} \\
\dot{\hat{h}}_{3}=-\gamma_{3} \operatorname{sgn}\left(h_{3}\right) \sigma_{3}\left(\ddot{\psi}_{d}-k_{3} \dot{\epsilon}_{\psi}\right)
\end{gathered}
$$

and

$$
U_{1}=\frac{1}{\cos x_{1} \cos x_{3}}\left[\hat{\lambda}_{9} x_{8}+\hat{m}\left(g+\ddot{z}_{d}-k_{4} \dot{\epsilon}_{z}-k^{\prime \prime \prime} \sigma_{4}\right)\right]
$$

with

$$
\begin{gathered}
\dot{\hat{\lambda}}_{9}=-\gamma_{4} \sigma_{4} x_{8} \\
\dot{\hat{m}}=-\gamma_{4} \sigma_{4}\left(\ddot{z}_{d}-k_{4} \dot{\epsilon}_{z}+g-k^{\prime \prime \prime} \sigma_{4}\right)
\end{gathered}
$$

where the sliding surfaces $\sigma_{2}, \sigma_{3}$ and $\sigma_{4}$ are such as:

$$
\begin{gathered}
\sigma_{2}=\dot{y}_{2}-\dot{\theta}_{d}-k_{2} \epsilon_{\theta} \\
\sigma_{3}=\dot{y}_{3}-\dot{\psi}_{d}-k_{3} \epsilon_{\psi} \\
\sigma_{4}=\dot{y}_{4}-\dot{z}_{d}-k_{4} \epsilon_{z}
\end{gathered}
$$

and $\epsilon_{\theta}, \epsilon_{\psi}$ and $\epsilon_{z}$ denote the tracking errors related to the pitch, heading and altitude, respectively:

$$
\begin{aligned}
\epsilon_{\theta} & =\theta_{d}-\theta \\
\epsilon_{\psi} & =\psi_{d}-\psi \\
\epsilon_{z} & =z_{d}-z
\end{aligned}
$$

Adopted control strategy is shown in the Fig. 2 below:

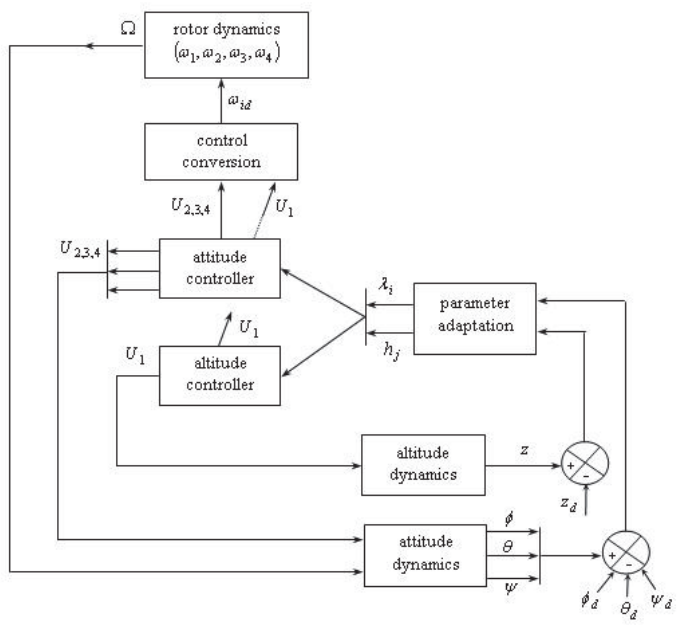

Fig. 2. Adopted Control Strategy

\section{Numerical Simulation}

The simulation results are obtained based on the real parameters in Table. 1 shown below.

1. Results without perturbation are shown in Fig. 3 to Fig. 10.

2. Flight with parametric uncertainties:

Two cases are treated. First, for an uncertainty of $20 \%$ on $m$ and $10 \%$ on $\left(I_{x}, I_{y}, I_{z}\right)$. After, for an uncertainty of $35 \%$ on $m$ and $15 \%$ on $\left(I_{x}, I_{y}, I_{z}\right)$. Desired trajectories tracking and parameters estimation are shown below (Fig. 11 to Fig. 14): 


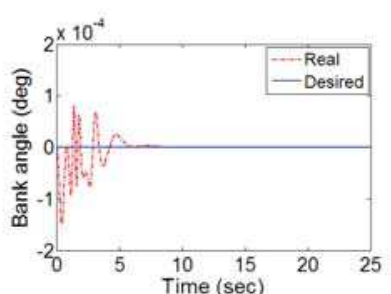

(a)

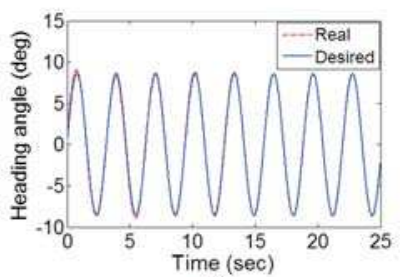

(c)

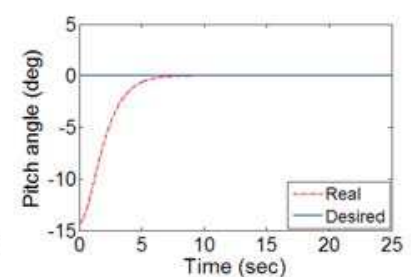

(b)

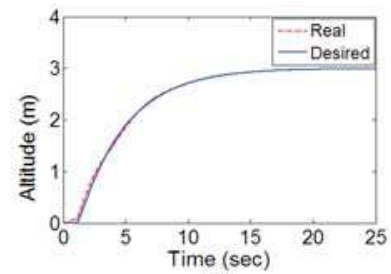

(d)
Fig. 3. Desired trajectories tracking related to the bank, pitch, heading angles and altitude, respectively
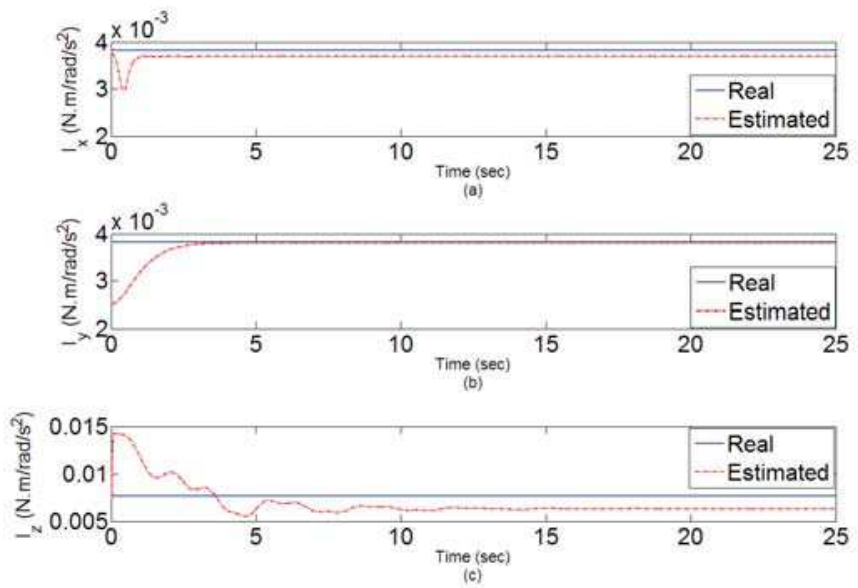

Fig. 4. Simulation results of inertial moments estimation
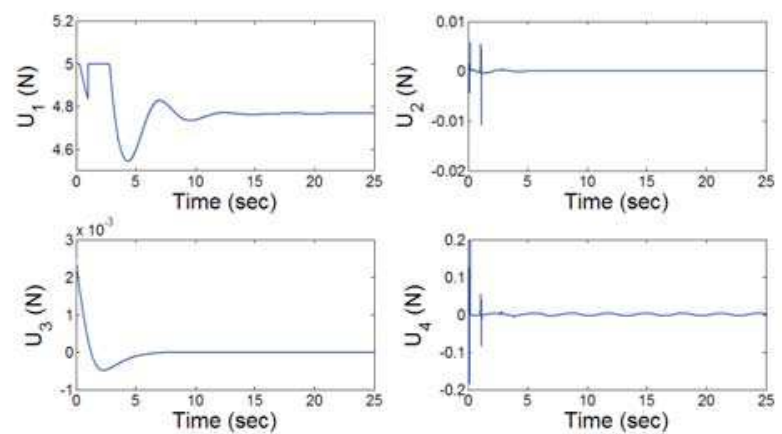

Fig. 5. Control inputs

\section{CONClusion}

In this paper, a direct adaptive sliding mode control have been developed in order to stabilize roll and pitch motions while tracking heading and altitude trajectories of a quadrotor.

During simulations, two cases are treated. the First one is considered for an uncertainty of $20 \%$ on the quadrotor mass
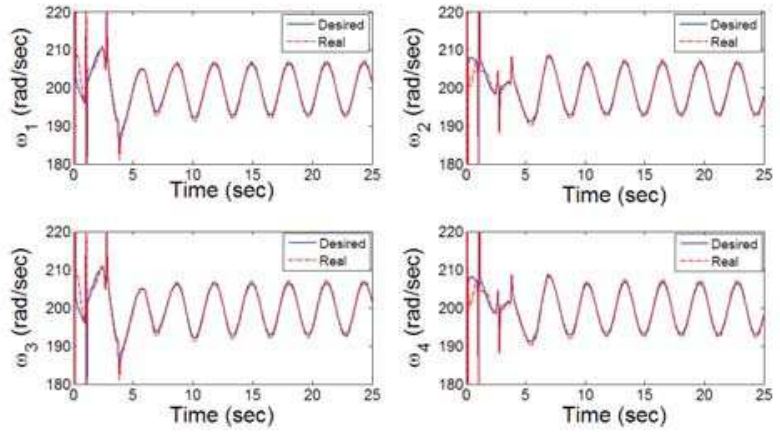

Fig. 6. Desired rotors velocities tracking
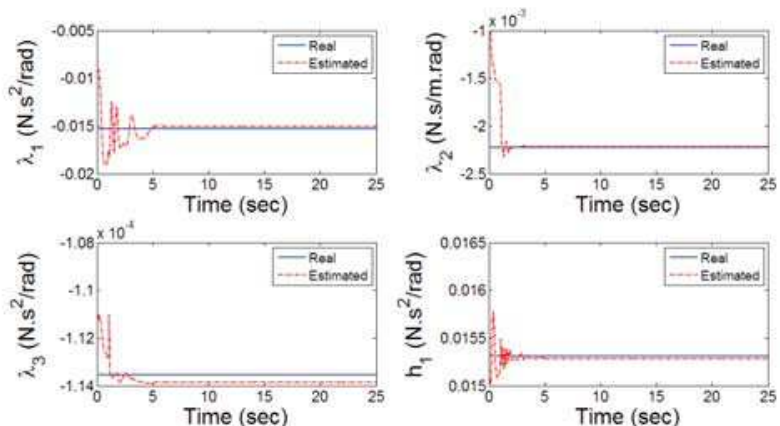

Fig. 7. Bank controller parameters estimation
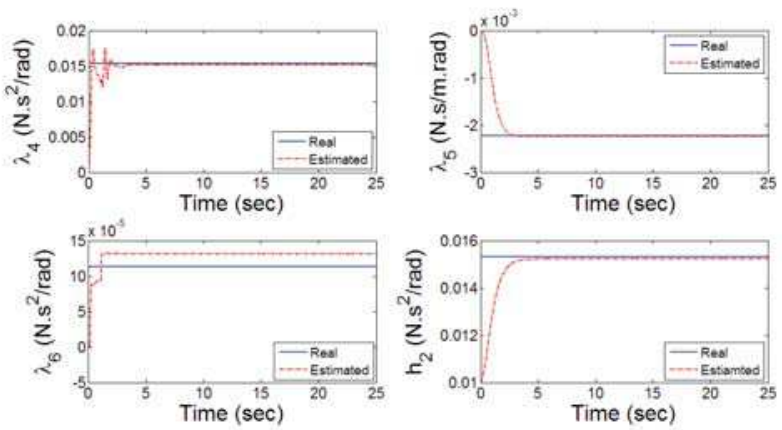

Fig. 8. Pitch controller parameters estimation

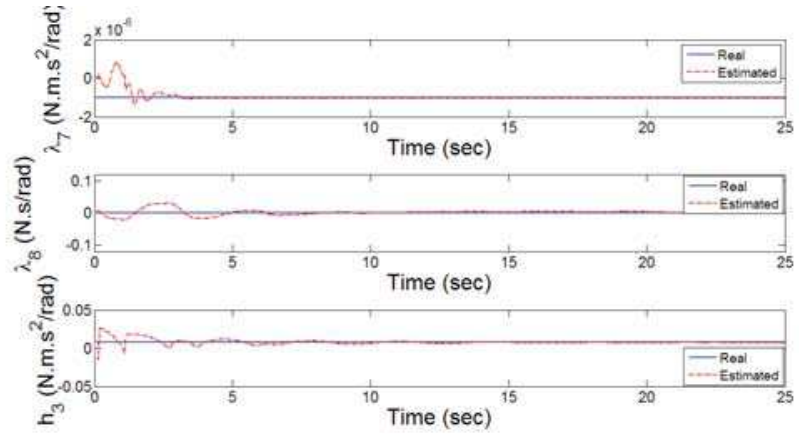

Fig. 9. Heading controller parameters estimation

$m$ and $10 \%$ on the inertia matrix $\operatorname{diag}\left(I_{x}, I_{y}, I_{z}\right)$ while the second case is for an uncertainty of $35 \%$ on $m$ and $15 \%$ on inertia matrix. Both of them are subject to an additional 

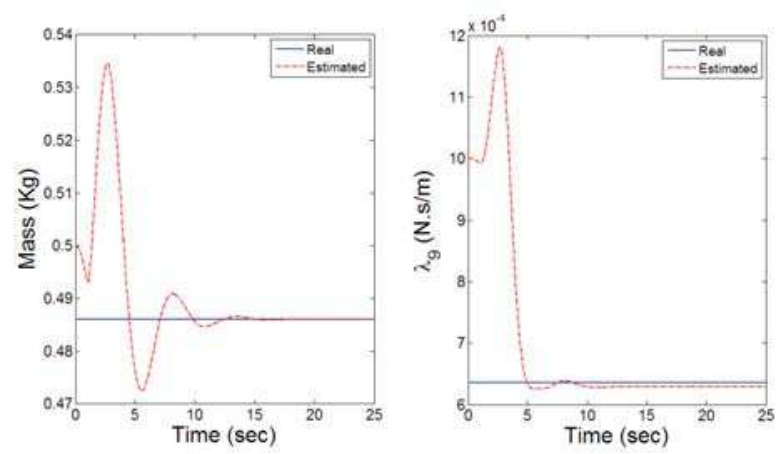

Fig. 10. Altitude controller parameters estimation
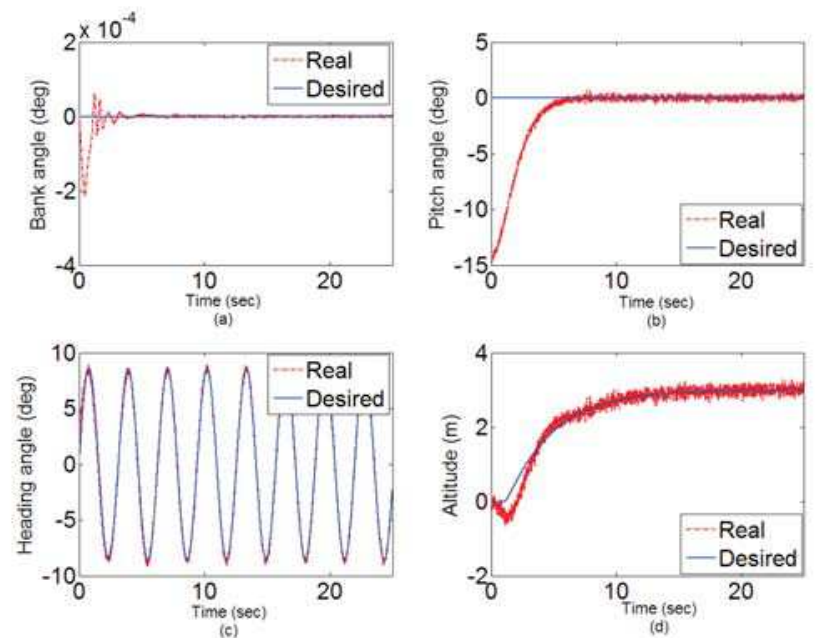

Fig. 11. Desired trajectories tracking for uncertainties of $20 \%$ on $m$ and $10 \%$ on $\left(I_{x}, I_{y}, I_{z}\right)$
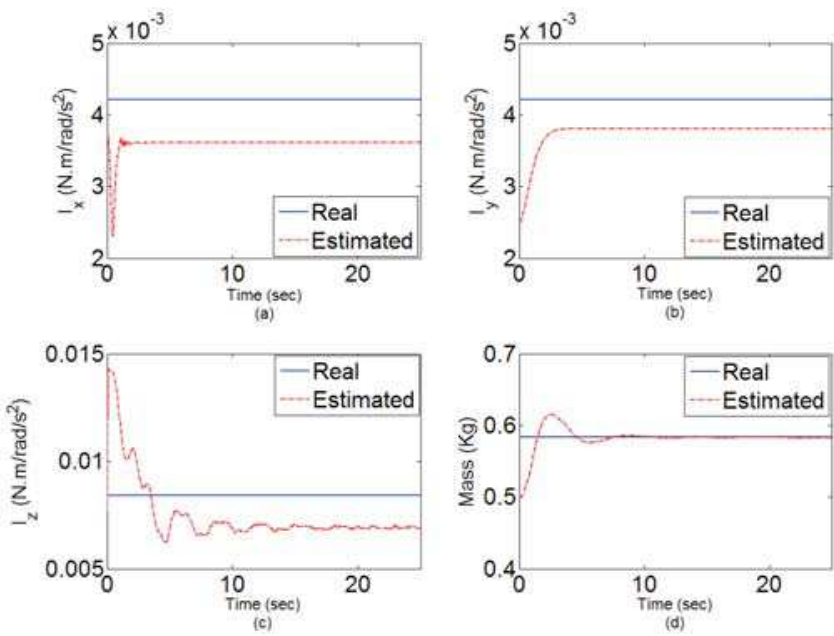

Fig. 12. Inertia matrix and mass estimation for uncertainties of $20 \%$ on $m$ and $10 \%$ on $\left(I_{x}, I_{y}, I_{z}\right)$

centered white gaussian noise.

Controller parameters estimation is based on the positivity and Lyapunov design principle. Considered unknown coefficients are related to the aerodynamic torques, inertia matrix and mass, respectively.
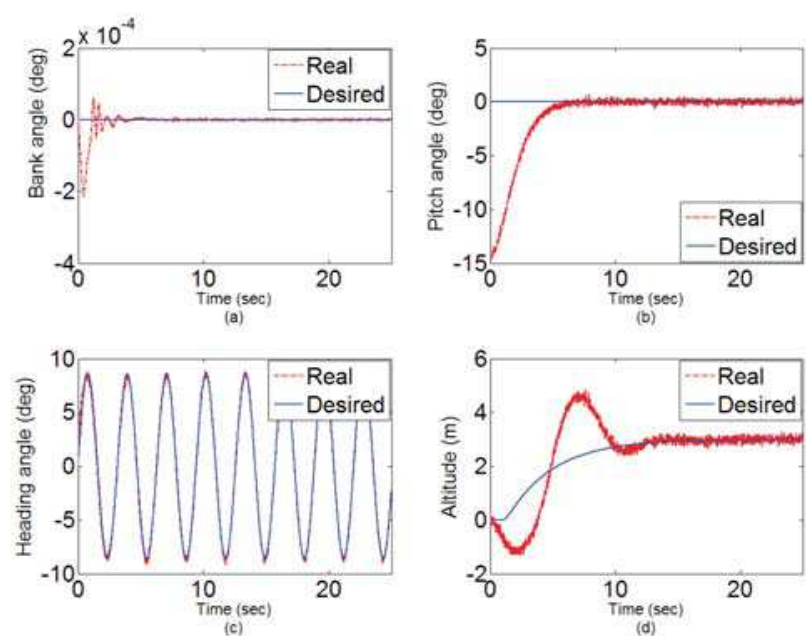

Fig. 13. Desired trajectories tracking for uncertainties of $35 \%$ on $\mathrm{m}$ and $15 \%$ on $\left(I_{x}, I_{y}, I_{z}\right)$
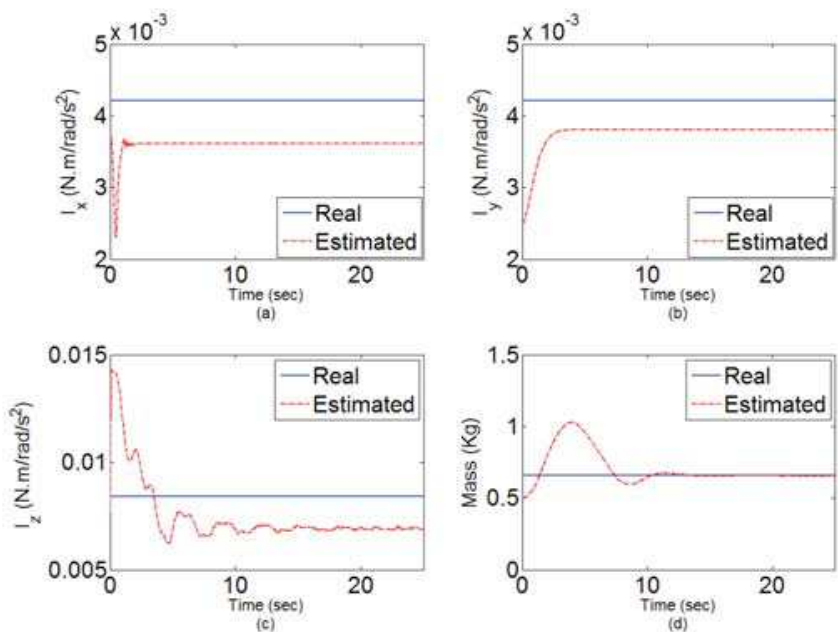

Fig. 14. Inertia matrix and mass estimation for uncertainties of $35 \%$ on $m$ and $15 \%$ on $\left(I_{x}, I_{y}, I_{z}\right)$

The effectiveness of the adaptive control laws have been evaluated by numerical simulations, showing good convergence results.

Further study will focus in a full state control of the quadrotor engaged in complex trajectory tracking.

\section{REFERENCES}

[1] H. Bouadi, M. Bouchoucha, and M. Tadjine, Modelling and Stabilizing Control Laws Design Based on Backstepping for an UAV TypeQuadrotor, in Proc. 6th IFAC Symposium on IAV, Toulouse, France, 2007.

[2] M. Bouchoucha, M. Tadjine, A. Tayebi, and P. Mllhaupt, Step by step robust nonlinear PI for attitude stabilization of four-rotor mini-aircraft, in Proc. 16th Mediterranean Conference on Control and Automation, Ajaccio, France, 2008.

[3] Y. Bouktir, M. Haddad, and T. Chettibi, Trajectory planning for a quadrotor helicopter, in Proc. 16th Mediterranean Conference on Control and Automation, Ajaccio, France, 2008

[4] A. Drouin, A. Brando Ramos, and F. Mora-Camino, Rotorcraft trajectory tracking by non linear inverse control, in Proc. 6th Brazilian Conference on Dynamics, Control and their Applications, Dincon 2007, Brazil, 2007. 
TABLE I

Quadrotor General Parameters

\begin{tabular}{c||c}
\hline Parameter & Value \\
\hline \hline Mass $(\mathrm{Kg})$ & 0.486 \\
Distance $d(\mathrm{~m})$ & 0.25 \\
$I_{x}\left(\mathrm{~N} . \mathrm{m} / \mathrm{rad} / \mathrm{s}^{2}\right)$ & $3.8278 \times 10^{-3}$ \\
$I_{y}\left(\mathrm{~N} . \mathrm{m} / \mathrm{rad} / \mathrm{s}^{2}\right)$ & $3.8278 \times 10^{-3}$ \\
$I_{z}\left(\mathrm{~N} . \mathrm{m} / \mathrm{rad} / \mathrm{s}^{2}\right)$ & $7.6566 \times 10^{-3}$ \\
$K_{f a x}(\mathrm{~N} / \mathrm{rad} / \mathrm{s})$ & $5.5670 \times 10^{-4}$ \\
$K_{f a y}(\mathrm{~N} / \mathrm{rad} / \mathrm{s})$ & $5.5670 \times 10^{-4}$ \\
$K_{f a z}(\mathrm{~N} / \mathrm{rad} / \mathrm{s})$ & $6.3540 \times 10^{-4}$ \\
$K_{f d x}(\mathrm{~N} / \mathrm{m} / \mathrm{s})$ & $5.5670 \times 10^{-4}$ \\
$K_{f d y}(\mathrm{~N} / \mathrm{m} / \mathrm{s})$ & $5.5670 \times 10^{-4}$ \\
$K_{f d z}(\mathrm{~N} / \mathrm{m} / \mathrm{s})$ & $6.3540 \times 10^{-4}$ \\
Lift force coefficient $K_{p}(\mathrm{~N} . \mathrm{m} / \mathrm{rad} / \mathrm{s})$ & $2.9842 \times 10^{-5}$ \\
Drag force coefficient $C_{D}(\mathrm{~N} . \mathrm{m} / \mathrm{rad} / \mathrm{s})$ & $3.2320 \times 10^{-7}$ \\
Rotor inertia $J_{r}\left(\mathrm{~N} . \mathrm{m} / \mathrm{rad} / \mathrm{s}^{2}\right)$ & $2.8385 \times 10^{-5}$ \\
$\beta_{0}$ & 189.63 \\
$\beta_{1}$ & 6.0612 \\
$\beta_{2}$ & 0.0122 \\
$b$ & 280.19 \\
\hline
\end{tabular}

[5] C. Nicol, C. J. B. Macnab, and A. Ramirez-Serrano, Robust neural network control of a quadrotor helicopter, IEEE, 2008.

[6] M. B. Srikanth, Z. T. Dydek, A. M. Annaswamy, and E. Lavretsky, A robust environment for simulation and testing of adaptive control for miniUAVs, in Proc. American Control Conference, St. Louis, USA, 2009.

[7] M. Huang, B. Xian, C. Diao, K. Yang, and Y. Feng, Adaptive tracking control of underactuated quadrotor unmanned aerial vehicles via backstepping, in Proc. American Control Conference, Baltimore, USA, 2010.

[8] H. Bouadi, Analysis and development of adaptive control techniques applied to flight control, M. S. thesis, Air Transportation Department, LARA, ENAC, France, 2010.

[9] I. Kanellakopoulos, P. V. Kokotovic and A. S. Morse, Systematic design of adaptive controllers for feedback linearizable systems, IEEE transactions on automatic control, vol. 36, No. 11, November 1991.

[10] M. Krstic, I. Kanellakopoulos and P. Kokotovic, Nonlinear and adaptive control design. John Wiley and Sons, Inc. 1995.

[11] R. Marino, P. Tomei, I. Kanellakopoulos and P. V. Kokotovic, Adaptive Tracking for a Class of Feedback Linearizable Systems, Proceeding of the $32^{\text {nd }}$ Conference on Decision and Control, San Antonio, Texas, December 1993.

[12] J. Slotine and L. Weiping, Applied nonlinear control. Prentice Hall, Upper Saddle River, NJ, 1991. 